\title{
SAMPLING AND RECONSTRUCTING DIFFUSION FIELDS IN PRESENCE OF ALIASING
}

\author{
Juri Ranieri, Martin Vetterli \\ School of Computer and Communication Sciences, \\ Ecole Polytechnique Fédérale de Lausanne (EPFL), CH-1015 Lausanne, Switzerland \\ E-mail: juri.ranieri@epfl.ch
}

\begin{abstract}
The reconstruction of a diffusion field, such as temperature, from samples collected by a sensor network is a classical inverse problem and it is known to be ill-conditioned. Previous work considered source models, such as sparse sources, to regularize the solution. Here, we consider uniform spatial sampling and reconstruction by classical interpolation techniques for those scenarios where the spatial sparsity of the sources is not realistic. We show that even if the spatial bandwidth of the field is infinite, we can exploit the natural lowpass filter given by the diffusion phenomenon to bound the aliasing error.
\end{abstract}

Index Terms- Diffusion equation, initial inverse problems, spatial sampling, aliasing error, interpolation.

\section{INTRODUCTION}

Sensor networks are often deployed to sense a phenomenon that can be described by a physical model. Knowing this model may allow a reduction of the quantity of measurements or an improvement of their quality. However this approach raises many challenges. For example, the theory of multidimensional sampling assumes homogeneous and interchangeable dimensions but for most physical fields, space and time are not equivalent.

In this paper, we assume that the sensor network is sensing a diffusive field, that is a solution of the following partial differential equation (PDE)

$$
\frac{\partial f(x, t)}{\partial t}-\frac{\partial^{2} f(x, t)}{\partial x^{2}}=g(x, t)
$$

where $f(x, t)$ is the field ${ }^{1}$ and $g(x, t)$ represents the source of diffusive material. The field is uniquely defined by (1) and the opportune boundary conditions, such as the value of the initial distribution $f(x, 0)$ or the value at certain locations.

Even if the mathematical model of a diffusive field is rather simple, it can model efficiently many real world scenarios: temperature in solid bodies, pollution dispersion [3],

\footnotetext{
This work was supported by an ERC Advanced Grant - Support for Frontier Research - SPARSAM Nr: 247006.

${ }^{1}$ Note that the diffusivity coefficient $\gamma$ is unitary for simplicity's sake. For a different $\gamma$, we consider a scaling of the temporal axis, $t^{\prime}=\gamma t$.
}
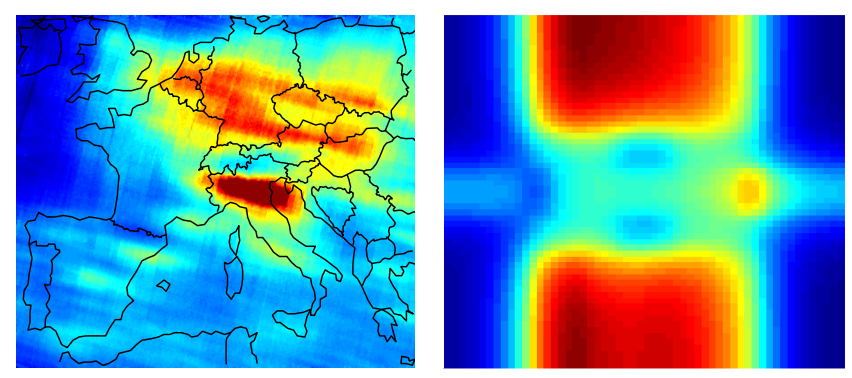

Fig. 1. Examples of diffusive fields. Left: the NO2 concentration over Europe on a weekday during winter [1]. Right: the temperature distribution in a commercial 8 core processor [2]. Note that the sources of these two fields are not sparse.

atmospheric plumes [4], and temperature distribution in a server room [5], to name a few. Recently, the temperature estimation in solid bodies showed its importance for thermal management of multi-core processors [2].

These applications often require the solution of an inverse problem. Generally speaking, an inverse problem is the inference of certain properties of the field, such as the initial distribution or the actual distribution, from a limited number of observations. The first known inverse problem of the diffusion equation goes back to Fourier and Kelvin [6], who tried to estimate the initial temperature of the Earth from the current temperature distribution. Unfortunately, it is known that many inverse problems involving the diffusion equation are severely ill-conditioned [7]. Recent works considered the spatial sparsity of the initial distribution as a regularization techniques. For example, Nehorai et al. [8] studied the detection and the localization of a single vapor-emitting source by a maximum likelihood estimator. Lu and Vetterli introduced two different approaches to the reconstruction of a sparse source distributions driving the diffusion field based on spatial super-resolution [9], and on an adaptive spatio-temporal sampling scheme [10]. An approach using compressed sensing on a discrete grid was proposed by Ranieri et al. [11] and it was generalized to the real line by Dokmanić et al. [12].

Unfortunately, in some practical scenarios we are often interested in recovering the actual field and not the source term and/or the spatial sparsity assumption for the initial distribution is not realistic. For example, when monitoring the temperature of a processor we would like to recover the actual thermal map and the temperatures sources are distributed, see 


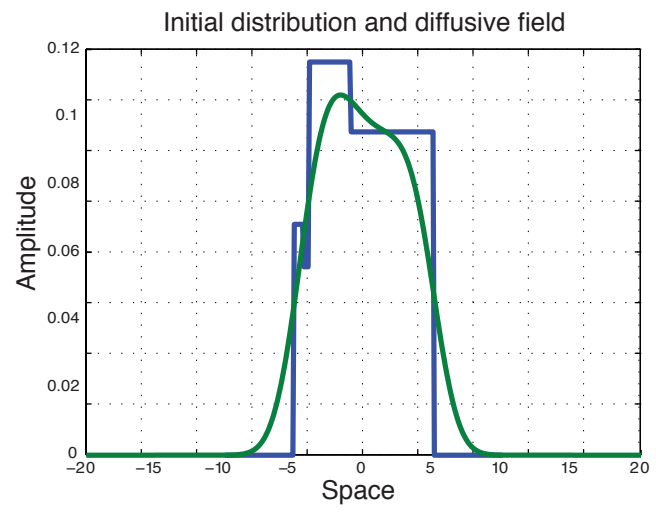

(a)

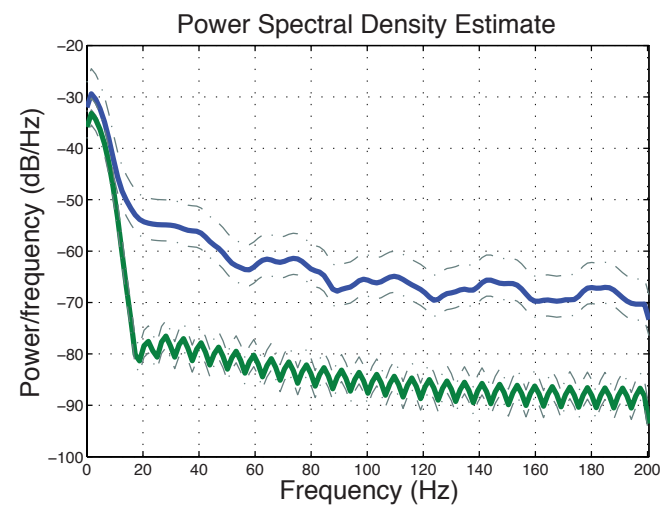

(b)

Fig. 2. (a) An initial distribution $f(x, 0)$ (blue) and the diffusive field $f\left(x, t_{0}\right)$ (green) at a certain time $t_{0}$. (b) The power spectrum of the two fields with the respective confidence interval. Note the significant decrease of power for the frequencies above $20 \mathrm{~Hz}$ for the diffusive field, leading to a significant reduction of the aliasing error.

Figure 1. A solution based on a finite elements has been introduced for the Poisson PDE by van Waterschoot et al. in [13], while a distributed algorithm has been proposed in [14].

Motivated by these considerations, we consider the following question: "how many spatial measurements should be collected to reconstruct exactly a diffusion field at a given time $t$ ?". We propose the following setup: the diffusive field is uniformly sampled in space and we recover the field using a classic result, the Shannon's sampling theorem [15]. The famous theorem states that a signal $f(t)$ with bandwidth $B$ is described completely by a set of its samples $\{f(n T)\}_{n}$, where $T \leq \frac{1}{2 B}$. If the signal is not bandlimited, a proper lowpass filter should be considered ahead of sampling to avoid aliasing error. However, if we try to apply this theory to spatial sampling of diffusive fields we may face two problems:

- The spatial bandwidth is infinite. In fact, given a field $f\left(x, t_{0}\right)$ at a fixed time instant $t_{0}$ the support of the Fourier transform (FT) $\mathcal{F}\left[f\left(x, t_{0}\right)\right]$ is not finite,

- It is physically impossible to apply a spatial low-pass filter on the field before sampling. Therefore, it is reasonable to expect a significant aliasing error.

In Section 2, we show that even if the bandwidth of any diffusive field is theoretically infinite, the magnitude of the FT decays sufficiently fast. In Section 3, we derive upper bounds for the aliasing error. We conclude the paper with numerical results to support our theoretical findings.

\section{SPATIAL BANDWIDTH OF A DIFFUSIVE FIELD}

The study of the spatial bandwidth of a diffusion field is complicated by the fact that the bandwidth changes over time. In what follows, we consider three simplified scenarios:

- a point source appearing at a known time,

- a generic distribution appearing at a known time,

- a spatially localized time-varying source.

\subsection{A Point Source}

Let us assume that at $t=0$ we have a Dirac's delta in $x=0^{2}$, that is $f(x, 0)=\delta(x)$ and that this source diffuses as time passes on an infinite domain. It is well known that the field evolves as

$$
f(x, t)=k(x, t)=\frac{1}{\sqrt{4 \pi t}} \exp \left\{\frac{-x^{2}}{4 t}\right\} .
$$

The solution is also known as the kernel $k(x, t)$, or alternatively the Green's function, of the diffusion equation and has a fundamental importance in determining the solution of diffusion equations with more complicated source terms. The FT of (2) for $t>0$ is

$$
\widehat{k}(\omega, t)=\exp \left\{-t \omega^{2}\right\},
$$

where $\omega$ are the spatial frequencies and the hat indicates the FT as in the rest of the paper. If we define the bandwidth as the difference between the upper and the lower frequencies of the set where (3) is different from zero, we have an infinite bandwidth. However, the magnitude decreases exponentially fast with $\omega^{2}$ and $t$.

\subsection{An initial distribution}

If we consider an initial distribution $f(x, 0)$ that diffuses in free space, the field is the convolution between the initial distribution and the kernel given in (2). Therefore, in the Fourier domain we have the following multiplication,

$$
\widehat{f}(\omega, t)=\widehat{f}(\omega, 0) \widehat{k}(\omega, t) .
$$

Given the Gaussian profile of $\widehat{k}(\omega, t)$ and the asymptotic behavior of $\widehat{f}(\omega, 0)$, see the Riemann-Lebesgue lemma [16], the

\footnotetext{
${ }^{2}$ Note that a different source location does not influence the magnitude of the FT of the field, being just a constant phase term.
} 
bandwidth of $\widehat{f}(\omega, t)$ is equivalent to the one of the initial distribution $\widehat{f}(\omega, 0)$. Moreover, the spectrum is increasingly low-passed with $t$ until the field has constant value at $t \rightarrow \infty$. See Figure 2 for an example of initial distribution smoothed by the diffusion kernel and the respective spectrums.

\subsection{A Time-varying Source}

Now, we assume that the source $g(x, t)$ of the field is spatially located at $x=0$ and its amplitude is time-varying, that is

$$
g(x, t)=\delta(x) g(t)
$$

The source is diffusing on an infinite domain and we assume that it is diffusing since $t=-\infty$. According to the theory of linear PDEs, the field $f(x, t)$ evolves in time and space as,

$$
f(x, t)=\int_{-\infty}^{t} \int_{-\infty}^{\infty} k(x-s, t-\tau) g(s, \tau) d s d \tau
$$

which is the convolution in space and time with the diffusion kernel given in (2). First, we consider the spatial integral and we exploit the property of the Dirac's delta,

$$
f(x, t)=\int_{-\infty}^{t} k(x, t-\tau) g(\tau) d \tau .
$$

Then, we look at the spatial FT to compute the spatial bandwidth of the diffusive field,

$$
\begin{aligned}
& \widehat{f}(\omega, t)=\int_{\mathbb{R}} f(x, t) \exp \{-\mathrm{i} \omega x\} d x \\
& =\int_{-\infty}^{t} \frac{1}{\sqrt{4 \pi(\tau-t)}} g(\tau) \int_{\mathbb{R}} \exp \left\{-\frac{x^{2}}{4(t-\tau)}-\mathrm{i} \omega x\right\} d x d \tau \\
& =\exp \left\{-t \omega^{2}\right\} \int_{-\infty}^{t} \exp \left\{\tau \omega^{2}\right\} g(\tau) d \tau
\end{aligned}
$$

More information can be obtained if we consider particular functions $g(t)$. For example, if $g(t)=\delta(t)$ we obtain again (3). If we consider a sinusoid at a frequency $\omega_{0}$ as a source term, that is

$$
g\left(t, \omega_{0}\right)=\sin \left(w_{0} t\right)
$$

then the diffusive field induced by a sinusoid evolves with $t$ in the Fourier domain as

$$
\widehat{f}\left(\omega, \omega_{0}, t\right)=\frac{\omega^{2} \sin \left(\omega_{0} t\right)-\omega_{0} \cos \left(\omega_{0} t\right)}{\omega^{4}+\omega_{0}^{2}} .
$$

We may remove the time-dependency of (7)

$$
\widehat{f}\left(\omega, \omega_{0}\right) \leq \max _{t} \widehat{f}\left(\omega, \omega_{0}, t\right)=\frac{1}{\sqrt{\omega^{4}+\omega_{0}^{2}}} .
$$

From this analysis of the FT of diffusive fields, we would like to underline an important aspect. The diffusive phenomena is characterized by the $\exp \left\{-t \omega^{2}\right\}$ term. It acts as a natu- ral low-pass filter, whose cutoff frequency is decreasing with time. Therefore, we can expect to sample and successfully reconstruct a diffusive field with a bounded aliasing error. We study this aspect in the following section.

\section{SAMPLING AND RECONSTRUCTION USING SHANNON'S THEOREM}

In this section, we study the sampling and the reconstruction of diffusive fields using Shannon's theorem. In particular, we are interested in the number of sensors that are needed to achieve a certain aliasing error.

Let us assume that we have a sensor network deployed with a spatial density of $2 \omega_{s}$ that spatially samples the diffusive field $f(x, t)$ at a given time $t$. The FT of the samples $\widehat{f}_{s}(\omega, t)$ is the periodic repetition of (3) with period $\omega_{s}$,

$$
\widehat{f}_{s}(\omega, t)=\sum_{k \in \mathbb{Z}} \widehat{f}\left(\omega-k \omega_{s}\right) .
$$

Let us define the aliasing error $\epsilon$ as the $\ell_{2}$-norm of the difference between $\widehat{f}(\omega, t)$ and $\widehat{f}_{s}(\omega, t)$ for $\omega \in\left\{-\frac{\omega_{s}}{2}, \frac{\omega_{s}}{2}\right\}$,

$$
\epsilon\left(\omega_{s}, t\right)=\int_{-\frac{\omega_{s}}{2}}^{\frac{\omega_{s}}{2}}\left|\widehat{f}(\omega, t)-\widehat{f}_{s}(\omega, t)\right|^{2} d \omega .
$$

Given the Gaussian profile of $\widehat{f}(\omega)$, we may expect that the aliasing error exponentially decreases with the sampling density $\omega_{s}$ and the time $t$. This intuition is confirmed by the following upper bound of the aliasing error.

Proposition 1. Let us consider a diffusive field generated by a point source appearing at $t_{0}=0$ spatially sampled at time $t$ with a density $2 \omega_{s}$. Even if the bandwidth of the field is infinite, the aliasing error can be upper bounded as:

$$
\epsilon\left(\omega_{s}, t\right) \leq \sqrt{\frac{\pi}{2 t}} \operatorname{Erfc}\left(\omega_{s} \sqrt{\frac{t}{2}}\right)
$$

Proof. First, we simplify the aliasing error using Cauchy Schwarz inequality and an opportune change of variable as,

$$
\begin{aligned}
\epsilon\left(\omega_{s}\right) & =\int_{-\frac{\omega_{s}}{2}}^{\frac{\omega_{s}}{2}}\left|\sum_{k \neq 0} \widehat{f}\left(\omega-k \omega_{s}\right)\right|^{2} d \omega \\
& \leq \sum_{k \neq 0} \int_{-\frac{\omega_{s}}{2}}^{\frac{\omega_{s}}{2}}\left|\widehat{f}\left(\omega-k \omega_{s}\right)\right|^{2} d \omega \\
& =\|F(\omega)\|^{2}-\int_{-\frac{\omega_{s}}{2}}^{\frac{\omega_{s}}{2}}|F(\omega)|^{2} d \omega,
\end{aligned}
$$

where we omitted the time dependency of the aliasing error for simplicity's sake. Then, we plug in the FT of the field 


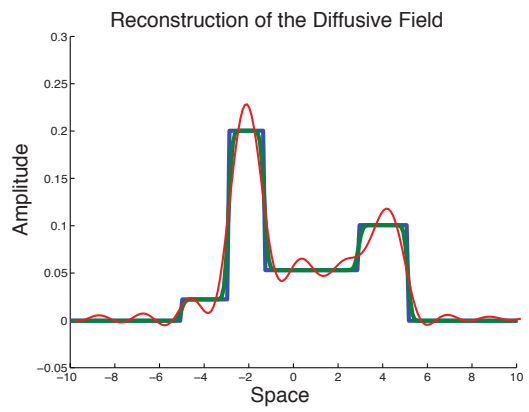

(a)

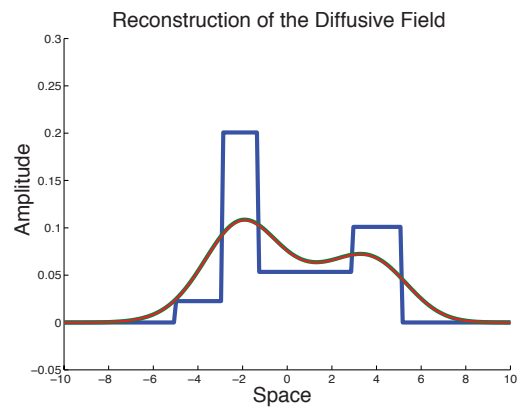

(b)

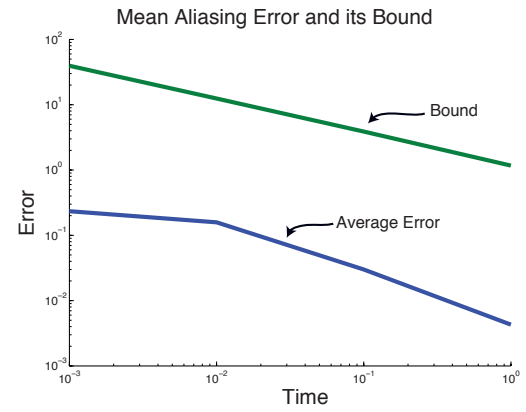

(c)

Fig. 3. Example of reconstructions at different times of a diffusive field generated by an initial distribution (blue). (a) The field (green) at $t=0.01$ and its reconstruction (red) from 25 samples. $\epsilon(0.01) \approx 0.199$ (b) The field (green) at $t=1$ and its reconstruction (red) from 25 samples, where $\epsilon(0.01) \approx 0.0039$. Note that in the second case the reconstruction error is significantly reduced due to the low-pass effect of the diffusion phenomena. (c) Comparison between the error bound computed in Proposition 1 and the average error computed over 100 random signals.

given in (3),

$\epsilon\left(\omega_{s}, t\right) \leq 2 \int_{\frac{\omega_{s}}{2}}^{\infty} \exp \left\{-2 \omega^{2} t\right\} d \omega=\sqrt{\frac{\pi}{2 t}} \operatorname{Erfc}\left(\omega_{s} \sqrt{\frac{t}{2}}\right)$,

where the complementary error function is defined as $\operatorname{Erfc}(x)=\int_{x}^{\infty} \exp \left\{-\omega^{2}\right\} d \omega$.

Since Erfc is upper bounded by a Gaussian, the aliasing error decays exponentially fast with $\omega_{s}$ and $t$. Therefore, even if the bandwidth is technically infinite, we can always sample a diffusion field and make the aliasing error as small as desired.

A similar result can be obtained for time-varying point sources defined in (5). When we consider a sinusoid at a frequency $\omega_{0}$, the diffusion field in the spatial Fourier domain is maximized as (8). This expression decays quickly w.r.t. $\omega^{2}$ and indicates that even if the bandwidth is theoretically infinite, we can again upper bound the aliasing error. This is subject of the following proposition.

Proposition 2. Let us consider a diffusive field generated by a single sinusoidal source located at $x=0$ with a frequency $\omega_{0}$, then the bandwidth of the field is infinite. However, the aliasing error, that is defined in (9), can be approximatively upper bounded as follows,

$$
\epsilon\left(\omega_{s}\right) \leq \frac{\pi-2 \arctan \left(\frac{\omega_{s}}{\sqrt{2 \omega_{0}}}\right)}{\sqrt{2 \omega_{0}^{3}}}
$$

where $\omega_{s}$ is the spatial sampling frequency.

Proof. Given the limited available space and the amount of computations necessary to show the result, we just sketch the proof. We plug the maximizer of $\widehat{f}\left(\omega, \omega_{0}\right)(8)$ into the aliasing error upper bound (10),

$$
\epsilon\left(\omega_{s}, \omega_{0}\right) \leq \frac{\pi}{\sqrt{2 \omega_{0}^{3}}}-\int_{-\frac{\omega_{s}}{2}}^{\frac{\omega_{s}}{2}} \frac{1}{w^{4}+w_{0}^{2}} d \omega
$$

The integral in (11) is lower bounded for $\omega_{s} \gg \omega_{0}$ as

$$
\int_{-\frac{\omega_{s}}{2}}^{\frac{\omega_{s}}{2}} \frac{1}{w^{4}+w_{0}^{2}} d \omega \geq \sqrt{\frac{2}{\omega_{0}^{3}}} \arctan \left(\frac{\omega_{s}}{\sqrt{2 \omega_{0}}}\right),
$$

proving the result.

\section{NUMERICAL RESULTS}

We present a series of numerical experiments showing the impact of time (and therefore of bandwidth) in the sampling and the reconstruction of diffusion fields. We choose a random initial distribution and we let it diffuse for a time $t$ before we sample it in space. We reconstruct the field using classical sinc interpolation. An example of the signals we consider and their reconstruction is given in Figure $3 a$ and $3 b$. Note that the reconstruction is more precise when $t$ is large because of the lower bandwidth. In Figure 3c, we show the average aliasing error for 100 random signals and the error bound given in Proposition 1. Being a bound for the worst case scenario, it is almost two orders of magnitude larger than the real aliasing error. Nonetheless, it follows the same decay.

\section{CONCLUSION}

We studied the problem of sampling and reconstructing diffusive field from a classical point of view: uniform spatial sampling followed by sinc interpolation. First, we obtained reliable characterizations of the spatial spectrum of diffusive fields generated by three different source types: point sources, distributions at known times and sinusoidal sources. Even if the bandwidth of the fields is infinite, the spectrum magnitude decays sufficiently fast to allow a faithful reconstruction. Therefore, we derived upper bounds for the aliasing error that show an exponential decay (or quadratic for timevarying sources) of the error w.r.t. a linear increase of the spatial sampling density. 


\section{REFERENCES}

[1] S Beirle, U Platt, M Wenig, and T Wagner, "Weekly cycle of NO2 by GOME measurements: a signature of anthropogenic sources," Atmospheric Chemistry and Physics, vol. 3, no. 6, pp. 2225-2232, 2003.

[2] J Ranieri, A Vincenzi, A Chebira, D Atienza, and M Vetterli, "EigenMaps: algorithms for optimal thermal maps extraction and sensor placement on multicore processors," in DAC, San Francisco, June 2012.

[3] A El Badia and T Ha-Duong, "An inverse problem in heat equation and application to pollution problem," Inverse and ill-Posed Problems, vol. 10, pp. 585-599, 2002.

[4] J M Stockie, "The mathematics of atmospheric dispersion modeling," SIAM Review, vol. 53, no. 2, pp. 349, 2011.

[5] K Chen, C E Bash, D M Auslander, and C D Patel, "Local temperature control in data center cooling: Part II, Statistical Analysis," Tech. Rep., 2006.

[6] H S Carslaw and J C Jaeger, Conduction of Heat in Solids, Oxford University Press, USA, 2 edition, 1986.

[7] C Hansen, Rank-Deficient and Discrete Ill-Posed Problems: Numerical Aspects of Linear Inversion, Soc. for Industrial Math., 1987.

[8] A Nehorai, B Porat, and E Paldi, "Detection and localization of vapor-emitting sources," IEEE Trans. Signal Process., vol. 43, no. 1, pp. 243-253, 1995.

[9] Y M Lu and M Vetterli, "Spatial super-resolution of a diffusion field by temporal oversampling in sensor net- works," in IEEE Int. Conf. on Acoustics, Speech and Signal Proc., Taiwan, 2009, pp. 2249-2252.

[10] Y M Lu and M Vetterli, "Distributed Spatio-Temporal Sampling of Diffusion Fields from Sparse Instantaneous Sources," in Proc. 3rd Int. Workshop on Comp Adv. in Multi-Sensor Adaptive Proc., 2009.

[11] J Ranieri, A Chebira, Y M Lu, and M Vetterli, "Sampling and reconstructing diffusion fields with localized sources," in IEEE Int. Conf. on Acoustics, Speech and Signal Proc., Prague, 2011, pp. 4016-4019, IEEE.

[12] J Ranieri, I Dokmanic, A Chebira, and M Vetterli, "Sampling and reconstruction of time-varying atmospheric emissions," in IEEE Int. Conf. on Acoustics, Speech and Signal Proc., Kyoto, 2012, pp. 3673-3676.

[13] T van Waterschoot and G Leus, "Static field estimation using a wireless sensor network based on the finite element method," Computational Advances in MultiSensor Adaptive Processing (CAMSAP), 2011 4th IEEE International Workshop on, pp. 369-372, 2011.

[14] T van Waterschoot and G Leus, "Distributed estimation of static fields in wireless sensor networks using the finite element method," in IEEE Int. Conf. on Acoustics, Speech and Signal Proc., Kyoto, 2012, pp. 2853-2856.

[15] C E Shannon, "Communication in the presence of noise," Proceedings of the IRE, vol. 37, no. 1, pp. 10-21, 1949.

[16] S Bochner and K Chandrasekharan, Fourier Transforms, Annals of Mathematics Studies. Princeton University Press, 1949. 